BNL-108469-2015-JA

\title{
Transmission X-ray Scattering as a Probe for Complex Liquid Surface Structures
}

\author{
Masafumi Fukuto, ${ }^{a, b *}$ Lin YAng, ${ }^{a}$ Dmytro NyKyPanchuK ${ }^{c}$ AND \\ IVAN KUZMENKO $^{d}$ \\ ${ }^{a}$ National Synchrotron Light Source II, Brookhaven National Laboratory, Upton, \\ New York 11973, USA, ${ }^{b}$ Condensed Matter Physics and Materials Science \\ Department, Brookhaven National Laboratory, Upton, New York 11973, USA, \\ ${ }^{c}$ Center for Functional Nanomaterials, Brookhaven National Laboratory, Upton, New \\ York 11973, USA, and ${ }^{d}$ Advanced Photon Source, Argonne National Laboratory, \\ Lemont, Illinois 60439,USA.E-mail: fukuto@bnl.gov
}

transmission X-ray scattering; liquid surface scattering; SAXS; WAXS; air/water interface

\begin{abstract}
The need for functional materials calls for increasing complexity in self-assembly systems. As a result, the ability to probe both local structure and heterogeneities, such as phase-coexistence and domain morphologies, has become increasingly important to controlling self-assembly processes, including those at liquid surfaces. The traditional x-ray scattering methods for liquid surfaces, such as specular reflectivity and grazing incidence diffraction, are not well suited to spatially resolving lateral heterogeneities due to large illuminated footprint. A possible alternative approach is to use scanning transmission x-ray scattering to simultaneously probe local intermolecular structures and heterogeneous domain morphologies on liquid surfaces. To test
\end{abstract}


the feasibility of this approach, we recently carried out transmission small- and wideangle x-ray scattering (TSAXS/TWAXS) studies of Langmuir films formed on water meniscus against a vertically immersed, hydrophilic Si substrate. First-order diffraction rings were observed in TSAXS patterns from a monolayer of hexagonally packed gold nanoparticles and in TWAXS patterns from a monolayer of fluorinated fatty acids, both as a Langmuir monolayer on water meniscus and as a Langmuir-Blodgett monolayer on the substrate. The patterns taken at multiple spots have been analyzed to extract the shape of the meniscus surface and the ordered-monolayer coverage as a function of the meniscus height. Our results, together with continual improvement in the brightness and spot size of x-ray beams available at synchrotron facilities, support the possibility of using scanning-probe TSAXS/TWAXS to characterize heterogeneous structures at liquid surfaces.

\section{Introduction}

Synchrotron x-ray scattering has greatly facilitated our understanding of liquid surface structure (Pershan \& Schlossman, 2012), having unraveled such phenomena as capillary wave fluctuations, surface-induced layering, surface freezing, the structural details of Langmuir and Gibbs monolayer phases, and ion distributions at aqueous interfaces (Als-Nielsen et al., 1982; Schwartz et al., 1990; Sanyal et al., 1991; Ocko et al., 1997; Kaganer et al., 1999; Fradin et al., 2000; Kuzmenko et al., 2001; Vaknin et al., 2003; Luo et al., 2006; Pershan, 2014). These pioneering studies demonstrate that synchrotron x-ray scattering is one of the few probes available that enable in situ structural characterization of liquid surfaces at interatomic and intermolecular length scales. The past decade has seen increasing attention to complex liquid surfaces, especially those involving self-assembly of nanoscale components such as lipids, surfactants, biomacromolecules, and inorganic nanoparticles. These nano-objects are 
assembled using liquid media, and liquid interfaces play a key role as a platform for promoting nucleation and growth. As the demand for functionality raises the complexity of self-assembly systems, it will become increasingly challenging to investigate these systems by relying solely on existing characterization methods.

One major challenge is to probe both local nanoscale structure and mesoscale heterogeneities, such as phase-coexistence and domain morphologies, which are common in two-dimensional (2D) assemblies of nano-objects at liquid interfaces (Kim et al., 2001; Tao et al., 2008; Wang et al., 1997; Ratanabanangkoon \& Gast, 2003; Fukuto et al., 2010; Kewalramani et al., 2011; Fukuto et al., 2013; Stanley et al., 2015). The established x-ray methods for characterizing liquid-surface structures, i.e., specular reflectivity and grazing incidence scattering, are not well suited to spatially resolving heterogeneities, since they are all based on illumination of surfaces at small incident angles (Pershan \& Schlossman, 2012) that result in a long illuminated footprint, typically of the order of a few $\mathrm{mm}$ to $100 \mathrm{~mm}$. In situ optical imaging of sub-mm liquid-surface domains is feasible only when the optical contrast in the heterogeneities of interest is either high enough (e.g., Brewster-angle microscopy) or enhanced by fluorescent labeling; and, even then, optical microscopy is rarely performed concurrently with x-ray structural measurements (Danauskas et al., 2007).

Another key challenge is to efficiently explore large parameter spaces. Typical multicomponent structures at liquid surfaces depend sensitively on thermodynamic, compositional and processing variables, including temperature, surface tension, constituent compositions at the interface as well as in the bulk liquid subphase. Again, the traditional x-ray scattering methods for liquid surfaces are not well suited to exploring vast parameter spaces since the requirement for large flat surface tends to result in large sample size and limits the sample throughput. ${ }^{\mathbf{1}}$ Throughput is severely limited for

\footnotetext{
${ }^{1}$ For example, typical x-ray measurements at air-water interfaces are based on preparing and measuring one sample at a time with aqueous solution volume of $\sim 100 \mathrm{~mL}$ to $1 \mathrm{~L}$ per sample.
}

IUCr macros version 2.1.6: 2014/10/01 
interfacial self-assembly systems that require long equilibration time or involve new materials with limited sample quantities.

To help address these challenges, the present study explores the potential of transmission x-ray scattering (TXS) as an alternative probe for liquid surface structures, complementary to the established reflection-mode methods. In TXS, the x-ray beam strikes the liquid-vapor interface at or near normal incidence and the scattered intensities are measured in the transmission mode. Previously, Narayanan et al. (Narayanan et al., 2004) observed TXS from a monolayer of nanoparticle arrays formed at the surface of an evaporating solution droplet. This pioneering study demonstrates that TXS can reveal a liquid-surface structure if the x-ray attenuation through the bulk liquid is not excessive and the background scattering in the reciprocal-space region of interest is sufficiently low. Under these favorable conditions, TXS should offer the following advantages over the grazing-incidence methods.

First, at normal incidence, the illuminated surface area is given by the transverse size of the x-ray beam. Continual improvement in the brightness and spot size of x-ray beams available at synchrotron facilities raises the possibility of implementing microbeam-based scanning transmission x-ray scattering microscopy (STXSM) for assemblies at liquid surfaces. By mapping out a diffraction feature of interest (e.g., peak intensity, lattice spacings, lattice orientations, etc.) as a function of the beam spot position on the surface, STXSM would enable simultaneous measurements of nanoscale packing structure and morphologies of microns-to-submillimeter scale domains at the interface. This imaging approach has already been demonstrated to be very useful for studies of fibers and biominerals (Paris, 2008; Seidel et al., 2008; Weaver et al., 2012; Kerschnitzki et al., 2013)

Second, because of the relatively small illuminated footprint, the area over which the liquid surface needs to be flat for TXS would be much smaller than required for IUCr macros version 2.1.6: 2014/10/01 
the grazing-incidence methods. This in turn implies that the liquid volume needed per sample could be substantially reduced for TXS, thus making it practical to handle an array of multiple samples at the same time to increase the sample throughput. Such a "high-throughput" approach has already been utilized in optical fluorescence microscopy studies of $2 \mathrm{D}$ protein crystallization at the air-water interface (Ratanabanangkoon \& Gast, 2003). Implementation of a similar sample-array approach for TXS should facilitate efficient exploration of complex liquid-surface structure in large parameter spaces.

To test the feasibility of using TXS as a position-sensitive probe for structures at liquid surfaces, we performed TXS measurements on Langmuir monolayers (LMs), formed on a water meniscus against a vertically immersed, hydrophilic Si substrate (70 $\mu \mathrm{m}$ thick). The experimental geometry is shown in Fig. 1. The water meniscus surface provides a well-defined air-water interface because of its known shape. Moreover, the nearly vertical orientation of the interface close to the meniscus edge, i.e., the threephase contact line, facilitates TXS measurements based on horizontal synchrotron x-ray beams. Using these sample and scattering configurations, we show that:

(i) TXS is a practical tool for characterizing ordered structures at liquid surfaces;

(ii) TXS combined with the scanning of the beam spot enables mapping of local structural information; and

(iii) TXS at non-normal incidence provides the ability to separate out structural information along the surface-parallel and surface-normal directions, a key advantage of the grazing incidence scattering methods.

For simplicity, we applied TXS to two systems of ordered LMs in which the meniscus surface and the surrounding horizontal air-water interface were uniformly coated by a $2 \mathrm{D}$ powder of hexagonally packed monolayer domains. The two systems exhibited order at very different length scales. The first system, chosen for high scattering IUCr macros version 2.1.6: 2014/10/01 
contrast, consisted of a monolayer of polystyrene-coated gold nanoparticles (AuNP) with an average core diameter of $9 \mathrm{~nm}$. Transmission small-angle x-ray scattering (TSAXS) measurements on AuNP monolayers revealed a first-order diffraction peak at the in-plane wave-vector transfer of $q_{x y}=0.07 \AA^{-1}$. As a step toward more weakly scattering systems, the second system was chosen to be a monolayer of a fluorinated fatty acid $\mathrm{CF}_{3}\left(\mathrm{CF}_{2}\right)_{12} \mathrm{COOH}(\mathrm{C} 14 \mathrm{~F})$. The $\mathrm{C} 14 \mathrm{~F}$ monolayer exhibited a first-order peak at $q_{x y}=1.25 \AA^{-1}$ in transmission wide-angle scattering (TWAXS) patterns. For both systems, the Langmuir-Blodgett (LB) transfer of the monolayer onto the vertical Si substrate allowed us to apply in situ TXS on both the LM at the meniscus-air interface and the transferred LB monolayer and to reveal variations in the monolayer coverage across the meniscus edge by scanning the beam spot vertically. Our results support the utility of TXS in characterizing heterogeneous monolayer structures on water at sub-mm resolutions, provided that care is taken to reduce the $\mathrm{x}$-ray path through the bulk subphase and minimize the background scattering from various sources.

\section{Background}

\subsection{Water meniscus against a hydrophilic wall}

Our experimental liquid surface consists of water menisci facing a vertically immersed, hydrophilic Si substrate (Fig. 1). For a liquid meniscus that completely wets a vertical wall (i.e., zero contact angle), its macroscopic shape is described by (de Gennes; F. Brochard-Wyart; \& Quere, 2004)

$$
y^{\prime}-y_{0}=\frac{h_{\max }}{\sqrt{2}}\left[\cosh ^{-1}\left(\frac{\sqrt{2} h_{\max }}{h}\right)-2\left(1-\frac{h^{2}}{2 h_{\max }^{2}}\right)^{1 / 2}\right],
$$

where $h=h\left(y^{\prime}\right)$ is the meniscus height relative to the horizontal liquid surface as

a function of the lateral distance $y^{\prime}$ away from the wall $\left(h \rightarrow 0\right.$ as $\left.\left|y^{\prime}\right| \rightarrow \infty\right)$. The constant $y_{0}$ in Eq. (1) is set by requiring $h=h_{\max }$ at $y^{\prime}=0$, i.e., at the wall. IUCr macros version 2.1.6: 2014/10/01 
The maximum liquid height $h_{\max }$ scales with the capillary length $\xi_{g}=\sqrt{\gamma /\left(\rho_{m} g\right)}$ as $h_{\max }=\sqrt{2} \xi_{g}$, where $\gamma$ and $\rho_{m}$ denote the liquid's macroscopic surface tension and mass density, respectively. For bare water surface at room temperature, $h_{\max }=3.8$ $\mathrm{mm}\left(\xi_{g}=2.7 \mathrm{~mm}\right)$ and $y_{0}=1.4 \mathrm{~mm}$. Figure 2 shows the relation between $h, y^{\prime}$, and the local surface tilt $\theta$ for the water meniscus, defined as $\theta \equiv 0$ for vertical surface. The local tilt and height of the water meniscus are related as (de Gennes; F. Brochard-Wyart; \& Quere, 2004)

$$
\sin (\theta)=1-\left(h / h_{\max }\right)^{2}
$$

where $h \leq h_{\max }$. Note that Eq. (2) satisfies $\theta=0$ at the wall $\left(h=h_{\max }\right)$ as required for complete wetting.

\subsection{X-ray kinematics}

Figure 1 illustrates the kinematics for x-ray scattering. In the lab frame, the $z^{\prime}$ axis is taken to be along the gravity axis, the horizontal liquid surface (far from a meniscus) lies in the $x^{\prime}-y^{\prime}$ plane, and the $y^{\prime}$ axis defines the direction of the horizontal beam that strikes our sample in transmission mode. The incident and scattered wave vectors are denoted as $\mathbf{k}_{\mathbf{i n}}=k \hat{\mathbf{y}}^{\prime}$ and $\mathbf{k}_{\text {out }}$, respectively, where $k=2 \pi / \lambda$ and $\lambda$ is the $\mathrm{x}$-ray wavelength. The wave-vector transfer is given by $\mathbf{q}=\mathbf{k}_{\mathbf{o u t}}-\mathbf{k}_{\mathbf{i n}}$. Any scattering direction can be defined in terms of two angles $(\psi, \phi)$, where $\psi$ is the angle between $\mathbf{k}_{\mathbf{i n}}$ and $\mathbf{k}_{\text {out }}$, so that $q=2 k \sin (\psi / 2)$, and $\phi$ is the azimuthal angle between the $x^{\prime}-z^{\prime}$ plane projection of $\mathbf{q}$ and the $z^{\prime}$ axis. The lab-frame components of $\mathbf{q}$ are given by

$$
\begin{aligned}
q_{x}^{\prime} & =k \sin (\psi) \sin (\phi) \\
& =q \sqrt{1-\zeta^{2}} \sin (\phi), \\
q_{y}^{\prime} & =-k[1-\cos (\psi)] \\
& =-q \zeta,
\end{aligned}
$$

IUCr macros version 2.1.6: 2014/10/01 


$$
\begin{aligned}
q_{z}^{\prime} & =k \sin (\psi) \cos (\phi) \\
& =q \sqrt{1-\zeta^{2}} \cos (\phi),
\end{aligned}
$$

where

$$
\zeta \equiv q / 2 k=\sin (\psi / 2)
$$

Diffraction patterns from 2D structures on an arbitrarily oriented surface will be more conveniently described in terms of the components of $\mathbf{q}$ that are defined relative to the local surface at the x-ray illuminated spot. We consider two simplest cases below that are directly relevant to our experiment. In the following, the $x-y$ plane is taken to be tangent to the local surface, and the $z$ axis represents the local surface normal.

2.2.1. Tilted surface: $\theta \neq 0$. We first consider a tilted surface whose local surface normal, the $z$ axis, lies in the $y^{\prime}-z^{\prime}$ plane and makes an angle $\theta$ to the $y^{\prime}$ axis, as shown in Fig. 1B. This configuration is relevant to Langmuir monolayers on a water meniscus that forms against a vertical substrate oriented normal to the incident beam. The limiting condition $\theta=0$ is relevant to transmission scattering from LB monolayers on a substrate at normal incidence. For this configuration, the surface-parallel and surface-normal components of $\mathbf{q}$ are given by

$$
\begin{gathered}
q_{x}=q_{x}^{\prime}=q \sqrt{1-\zeta^{2}} \sin (\phi), \\
q_{y}=-q\left[\zeta \sin (\theta)+\sqrt{1-\zeta^{2}} \cos (\phi) \cos (\theta)\right], \\
q_{z}=-q\left[\zeta \cos (\theta)-\sqrt{1-\zeta^{2}} \cos (\phi) \sin (\theta)\right] .
\end{gathered}
$$

Suppose that an x-ray illuminated spot on the tilted surface contains a powder of ordered 2D domains, as is the case for the Langmuir and LB monolayers in the present study. With $d$ denoting a periodic spacing for the $2 \mathrm{D}$ lattice, the corresponding diffraction peak would occur when the local surface-parallel component $q_{x y} \equiv \sqrt{q_{x}^{2}+q_{y}^{2}}$ IUCr macros version 2.1.6: 2014/10/01 
satisfies the Bragg condition

$$
q_{x y}=G \equiv 2 \pi / d .
$$

Using Eqs. (7), (8), and (10), the corresponding Bragg condition for the total wavevector transfer $q$ can be expressed as

$$
\begin{aligned}
(G / q)^{2}= & \left(1-\zeta^{2}\right)+\sin ^{2}(\theta)\left[\zeta^{2}-\left(1-\zeta^{2}\right) \cos ^{2}(\phi)\right] \\
& +\zeta \sqrt{1-\zeta^{2}} \cos (\phi) \sin (2 \theta) .
\end{aligned}
$$

If the scattering angle is sufficiently small such that $\zeta=\sin (\psi / 2) \ll 1$, as is the case in our experiments, Eq. (11) can be approximated as

$$
q \approx \frac{G}{\sqrt{1-\cos ^{2}(\phi) \sin ^{2}(\theta)}} .
$$

Thus, under the small-angle approximation, the Bragg peak in the equatorial plane $\phi= \pm \pi / 2$ occurs at $q \approx G$, regardless of the surface tilt $\theta$. Note that Eq. (12) reduces to $q \approx G / \sin (\phi)$ at grazing incidence $\theta \rightarrow \pi / 2$, consistent with a Bragg rod.

Transmission scattering measurements map out the $(q, \phi)$ space. Thus, Eq. (11), and Eq. (12) where applicable, describes the $\phi$-dependence of the Bragg condition for $q=2 k \zeta$ for a given set of $(G, \theta)$. Note that each Bragg-satisfying set $(q, \phi, \theta)$ is associated with a surface-normal component $q_{z}=\sqrt{q^{2}-G^{2}}$, which varies with $(q, \phi, \theta)$ according to Eq. (9).

It should be noted that our experimental approach (Fig. 1B) probes two surface spots, whose tilt angles are of the same magnitude but of opposite signs $( \pm \theta)$. Our measurements and analyses were carried out under conditions where scattering contributions from these two spots were practically equivalent. First, the measurements were carried out at large enough $h$, that is, the ratio of the inter-spot separation to the spot-detector distance was sufficiently small, that the $q$-resolution spread due to having two surface spots was smaller than the Bragg-peak broadening due to other IUCr macros version 2.1.6: 2014/10/01 
causes. Second, the analyses were performed only for cases where scattering occurred at or near the surface horizon $(\phi= \pm \pi / 2)$ or the small-angle approximation was valid $(\zeta \ll 1)$, such that both $q$ and $q_{z}$ became independent of the sign of $\theta$ [Eq. (9), (11), and (12)]. Finally, the scattering angle $\psi$ was small enough $[\cos (\psi) \approx 1]$ that the intensity attenuation through the bulk meniscus water and the substrate was essentially the same for the two scattering contributions.

2.2.2. Vertical, rotated surface: $\omega \neq 0$. Here we consider a vertical surface $(\theta=0)$ that deviates from the normal incidence condition due to rotation, by angle $\omega$, about the vertical $z^{\prime}$ axis in the lab frame, as illustrated in Fig. 1C. In this case, the surfacenormal $z$ axis lies in the $x^{\prime}-y^{\prime}$ plane and becomes parallel to the $y^{\prime}$ axis at $\omega=0$. As will be shown below, transmission scattering at $\omega \neq 0$ provides a means to extract the $q_{z}$ dependence of Bragg rods for vertical monolayers from a single scattering image. For this configuration, the components of $\mathbf{q}$ are given by

$$
\begin{gathered}
q_{x}=q\left[\zeta \sin (\omega)+\sqrt{1-\zeta^{2}} \sin (\phi) \cos (\omega)\right], \\
q_{y}=-q_{z}^{\prime}=-q \sqrt{1-\zeta^{2}} \cos (\phi), \\
q_{z}=-q\left[\zeta \cos (\omega)-\sqrt{1-\zeta^{2}} \sin (\phi) \sin (\omega)\right] .
\end{gathered}
$$

Again, for a powder of ordered 2D domains on the surface, the Bragg condition $\left(q_{x y}=G\right)$ is obtained when $q$ satisfies

$$
\begin{aligned}
(G / q)^{2}= & \left(1-\zeta^{2}\right)+\sin ^{2}(\omega)\left[\zeta^{2}-\left(1-\zeta^{2}\right) \sin ^{2}(\phi)\right] \\
& +\zeta \sqrt{1-\zeta^{2}} \sin (\phi) \sin (2 \omega)
\end{aligned}
$$

Under the small-angle approximation $(\zeta \ll 1)$, this reduces to

$$
q \approx \frac{G}{\sqrt{1-\sin ^{2}(\phi) \sin ^{2}(\omega)}}
$$

IUCr macros version 2.1.6: 2014/10/01 


\section{Experiment}

\subsection{Materials}

3.1.1. Polystyrene-capped gold nanoparticles (AuNP). An aqueous solution of citratestabilized gold nanoparticles of nominal diameter $10 \mathrm{~nm}$ was purchased from Ted Pella. Typical measured core diameter was $9 \mathrm{~nm}$ (Srivastava et al., 2014b; Srivastava et al., 2014a). The citrate-capped particles were transferred to toluene following a general procedure (Wang et al., 2010) and using oleyamine (OA) as a transfer agent. The excess of OA was removed by centrifugation at $10000 \mathrm{RCF}$ for $1.5 \mathrm{~h}$, followed by resuspension in a $10 \mathrm{mM}$ toluene solution of polystyrenethiol. Weakly bound OA is readily substituted by strongly binding polystyrenethiol (Hiramatsu \& Osterloh, 2004). The centrifugation was repeated and the supernantant was exchanged with a $1 \mathrm{mM}$ polystyrenethiol solution in toluene. The particles were incubated overnight. After the ligand exchange, the particles were purified three times by successive precipitation and re-suspension in toluene. After the final purification step, toluene was added to achieve a $1 \mu \mathrm{M}$ nominal particle concentration.

3.1.2. Fluorinated fatty acid $(C 14 F)$. Perfluorotetradecanoic acid $\mathrm{CF}_{3}\left(\mathrm{CF}_{2}\right)_{12} \mathrm{COOH}$ (Sigma-Aldrich, 96\% purity) was used as received. A spreading solution for the monolayer formation was prepared by dissolving C14F in a 20:1 chloroform/methanol (v/v) mixture at the concentration of $2.18 \mathrm{mg} / \mathrm{ml}$.

\subsection{Methods}

3.2.1. Monolayer formation. Monolayers on water was prepared using a teflon Langmuir trough equipped with a surface tension balance and a compression barrier. The trough was enclosed in an aluminum canister with Kapton x-ray windows. At the beamline, the trough assembly was mounted on the liquid-surface diffractometer via

IUCr macros version 2.1.6: 2014/10/01 
a set of vibration isolation stages at the sample position. Ultrapure water (Milli-Q, 18.2 $\mathrm{M} \Omega-\mathrm{cm}$ ) was used as subphase. Before spreading a monolayer, a Piranha-etched, hydrophilic silicon substrate $(10 \mathrm{~mm}$ wide $\times 25 \mathrm{~mm}$ high $\times 70 \mu \mathrm{m}$ thick $)$ was immersed vertically into the pure-water subphase using a manual micrometer coupled to the substrate holder. With the substrate in contact with the subphase, a spreading solution containing AuNP or $\mathrm{C} 14 \mathrm{~F}$ of known volume and concentration was spread on the surface drop by drop using a Hamilton syringe. Before starting x-ray measurements, the enclosure was filled with humidified He gas to minimize background scattering. The monolayer was compressed slightly and allowed to relax to nonzero surface pressure $(5-10 \mathrm{mN} / \mathrm{m})$, in order to ensure complete surface coverage.

The meniscus edge height depends on the surface pressure $\Pi$ as $h_{\max } \propto \sqrt{\gamma}=$ $\sqrt{\gamma_{w}-\Pi}$ (Sec. 2.1), where the water surface tension is $\gamma_{w}=72 \mathrm{mN} / \mathrm{m}$ at room temperature. The TSAXS images from AuNP monolayers were taken at $\Pi=7 \mathrm{mN} / \mathrm{m}$, corresponding to $h_{\max }=3.6 \mathrm{~mm}$ (also see the solid curve in Fig. 6B). The TWAXS images from $\mathrm{C} 14 \mathrm{~F}$ monolayers were taken at $\Pi=5 \mathrm{mN} / \mathrm{m}$, corresponding to $h_{\max }=$ $3.7 \mathrm{~mm}$.

3.2.2. X-ray scattering. Experiments on AuNP monolayers were carried out at Beamline X22B of the National Synchrotron Light Source at Brookhaven National Laboratory, using the liquid-surface diffractometer (x-ray energy $8.17 \mathrm{keV}$ ). The setups for control x-ray reflectivity (XR) and grazing-incidence small-angle x-ray scattering (GISAXS) measurements were similar to those used previously (Srivastava et al., 2014b; Srivastava et al., 2014a). For TSAXS, the incident x-ray beam was set to be horizontal, and the position of the x-ray illuminated spot on the meniscus or substrate surface was controlled by motorized vertical and lateral sample stages. The beam size and flux at the sample position was $0.1 \mathrm{~mm}$ high $\times 0.25 \mathrm{~mm}$ wide and $5 \times 10^{7}$ pho- 
tons/s, respectively. TSAXS images were captured using a CCD area detector (Princeton Instruments) located $1.0 \mathrm{~m}$ from the sample position. Typical exposure time was $300 \mathrm{~s}$, which was short enough that no discernible radiation effects could be seen in the TSAXS patterns.

Measurements on $\mathrm{C} 14 \mathrm{~F}$ monolayers were carried out at Beamline 9ID-C of the Advanced Photon Source at Argonne National Laboratory, using the liquid-surface diffractometer. The x-ray energy was $13.5 \mathrm{keV}$ for most measurements, except that the TWAXS measurements for extracting Bragg rods were taken at $22.1 \mathrm{keV}$. The setups for control XR and grazing-incidence x-ray diffraction (GIXD) measurements were described previously (Witte et al., 2010). For TWAXS, the diffractometer setup was similar to the TSAXS setup above, except that a motorized horizontal guardslit aperture and a motorized beamstop were installed inside the sample enclosure, immediately before and after the teflon Langmuir trough, respectively. The aperture and beamstop were used to minimize the background scattering from the entrance and exit Kapton windows of the trough enclosure, respectively. The beam size was $0.5 \mathrm{~mm}$ high $\times 0.4 \mathrm{~mm}$ wide at the sample position, and the flux was $1 \times 10^{12}$ photons $/ \mathrm{s}$ at $13.5 \mathrm{keV}$ and $2 \times 10^{11}$ photons $/ \mathrm{s}$ at $22.1 \mathrm{keV}$. TWAXS images were collected using a Pilatus 100K pixel-array detector (Dectris) located $0.20 \mathrm{~m}$ from the sample position. Typical exposure time of $120 \mathrm{~s}$ was used after ensuring that no radiation-dependent changes were evident in the TWAXS patterns.

\section{Results and Discussion}

\subsection{Monolayers of gold nanoparticles (AuNP)}

The formation of a AuNP monolayer at the air-water interface was first verified by control XR and GISAXS measurements (Fig. 3) on a horizontal part of the water surface, located far away $(>1 \mathrm{~cm})$ from the vertically immersed Si substrate. The 
measured XR data displays an intensity modulation with a period of $\Delta q_{z}=2 \pi / L=$ $0.06 \AA^{-1}$, signifying the presence of a layer with thickness $L=10 \mathrm{~nm}$. The comparison with the AuNP core diameter of $9 \mathrm{~nm}$ demonstrates that the layer consists of a single monolayer of AuNPs. The observed GISAXS pattern exhibits Bragg peaks at $q_{x y}=$ $G_{1}=0.070 \AA^{-1}$ and $q_{x y}=\sqrt{3} G_{1}=0.12 \AA^{-1}$, consistent with a hexagonally packed monolayer of AuNPs with a nearest-neighbor (NN) distance of $(2 / \sqrt{3}) 2 \pi / G_{1}=10.4$ nm.

With the same monolayer-coated surface still intact, a series of TSAXS patterns were collected from multiple spots along the substrate-supported meniscus surface, as a function of $h$ (i.e., the height relative to the horizontal surface, far from the meniscus).For these measurements, the incident x-ray beam was horizontal and penetrated through the vertically immersed Si substrate at normal incidence $(\omega=0)$. Typical images obtained are summarized in Fig. 4A. The TSAXS patterns observed from spots just below the meniscus edge $\left(h<h_{\max }\right)$ exhibited a clear first-order diffraction ring corresponding to the Bragg condition $q_{x y}=G_{1}=0.070 \AA^{-1}$ for the hexagonal packing of AuNPs in the monolayer. As the spot was lowered well below the meniscus edge, the observed Bragg ring became diminished in intensity. This is also demonstrated in Fig. 5C, in which the observed TSAXS intensity is plotted as a function of the total wave vector transfer $q$ along the horizon $\left(\phi=90^{\circ}\right)$ for various $h$. The intensity reduction is due to the increase in the x-ray path through bulk water, and hence increasing x-ray absorption, with decreasing $h$ (Fig. 5A). Finally, for spots above the meniscus edge $\left(h>h_{\max }\right)$, no diffraction ring was observed, consistent with the uncoated, bare substrate surface.

A set of TSAXS images were collected again after the monolayer was LB-deposited onto the vertical Si substrate by raising the substrate by $\Delta h \sim 4 \mathrm{~mm}$. As demonstrated in Fig. 4B, the TSAXS images from spots within the $\Delta h$-high strip above the meniscus

IUCr macros version 2.1.6: 2014/10/01 
edge now exhibited the Bragg ring, confirming the LB transfer of the AuNP monolayer onto the substrate. Ex-situ XR and GISAXS measurements of the dry AuNP-coated substrate (not shown) further confirmed that the transferred film remained monolayerthick and preserved the hexagonal packing of AuNPs.

The $h$-dependent TSAXS scans enabled us to extract position-dependent structural features, even for the uniform monolayer structure considered here. Two such examples, namely the meniscus shape and the surface coverage, are discussed separately below.

4.1.1. Meniscus shape: surface tilt vs. $h$. A notable feature of the TSAXS patterns (Fig. 4) is that the Bragg ring observed for $h<h_{\max }$ became vertically elongated as the x-ray spot was lowered along the meniscus. This elongation effect, captured in Eqs. (11) and (12), arises from the tilt of the air-meniscus interface, with the tilt angle $\theta$ increasing with decreasing $h$. A similar effect was observed previously by Narayanan et al. in the TSAXS patterns from AuNP monolayers formed on the curved surface of an evaporating solution droplet (Narayanan et al., 2004).

The sensitivity of the Bragg ring shape to the surface tilt is demonstrated in Fig. 5D. This plot shows radial intensity profiles $I(q)$ of a TSAXS pattern along selected detector-plane azimuthal directions $\{\phi\}$ (Fig. 5B). The plotted data was obtained from a spot at $h=3.0 \mathrm{~mm}$, where the surface was expected to be tilted at $\theta=18^{\circ}$ via Eq. (2). The elongation effect on the Bragg ring is clearly evident, and the peak shift to higher $q$ with decreasing $\phi$ is consistent with Eq. (12). The tilt effect becomes progressively prominent as the x-ray spot is lowered further along the meniscus. This is demonstrated in Fig. 6A (symbols), which plots the $\phi$-dependent variations of the radial intensity-peak position $q$ extracted from the observed TSAXS patterns for various $h$. The peak position for given $(h, \phi)$ was obtained by fitting the measured intensity

IUCr macros version 2.1.6: 2014/10/01 
profile $I(q)$ to a Gaussian with a linear background.

To extract the surface-tilt angle $\theta$, the $\phi$ dependence of the peak position $q$ for each value of $h$ was fitted using Eq. (12) with fixed $G=G_{1}$. The best-fits are shown as solid curves in Fig. 6A. The corresponding best-fit $\theta$ values are plotted as a function of $h$ in Fig. 6B (symbols). The $h$ dependence of the extracted surface tilts is compared with the predictions from Eq. (1) for bare water surface (dashed line) and for the measured surface pressure of $\Pi=7 \mathrm{mN} / \mathrm{m}$ for the AuNP monolayer (solid line). The good agreement between the extracted and predicted surface-tilt variations with $h$ demonstrates that the observed TSAXS scans are consistent with the expected shape of the meniscus. This result supports the utility of the meniscus as a well-defined air-water interface that can be exploited for quantitative TSAXS characterization of liquid surface structures.

4.1.2. Coverage by ordered domains vs. h. A measure of local surface coverage by monolayer domains is given by the peak intensity, $\Delta I_{\text {peak }}$, in the background-subtracted radial intensity profile $\Delta I(q)$ along the horizon, i.e., at $\phi= \pm 90^{\circ}$ (Fig. 5C). Note that the small-angle approximation applies here $(\zeta=0.0086 \ll 1$ at $q=G)$, and Eq. (9) reduces to $q_{z} \approx 0$ at $\phi= \pm 90^{\circ}$ for all $\theta \in[0, \pi / 2]$. With the $q_{z}$ dependence of $\Delta I(q)$ eliminated, the peak intensity $\Delta I_{\text {peak }}=\Delta I\left(q_{x y}=G\right)$ at the horizon should simply scale with the local areal coverage by the hexagonally packed AuNP monolayer at the given illuminated spot.

The monolayer coverages at different beam spots can be compared by making two $h$-dependent corrections to $\Delta I_{\text {peak }}$ for spots below the meniscus edge $\left(h<h_{\max }\right)$. One correction is for the x-ray absorption through bulk water: lowering the beam spot along the meniscus results in an increased x-ray path through water (Fig. 5A) and hence a reduction in transmitted intensity. The other correction is for the surface

IUCr macros version 2.1.6: 2014/10/01 
tilt variation along the meniscus: for a given beam size, increasing $\theta$ expands the illuminated footprint on the monolayer surface, leading to enhanced scattering. The two corrections have opposite effects on the measured intensity. Taking into account both of these effects, the surface coverage $C$ is proportional to:

$$
C\left(h<h_{\max }\right) \propto \frac{\exp (-2 \mu y)}{\cos (\theta)} \Delta I_{\text {peak }}
$$

and

$$
C\left(h \geq h_{\max }\right) \propto \Delta I_{\text {peak }},
$$

where

$$
\Delta I_{\text {peak }}=\Delta I\left(q_{x y}=G, q_{z}=0\right) .
$$

In Eq. (18), $2 y$ corresponds to the x-ray path length through water (Fig. 5A), $\mu^{-1}$ (= $1.06 \mathrm{~mm}$ for $8.17 \mathrm{keV}$ x-rays $)$ is the x-ray attenuation length for water, and $y$ and $\theta$ depend on $h$ according to Eqs. (1) and (2), respectively. To extract $\Delta I_{\text {peak }}$, the measured intensity profile $I(q)$ along the horizon $\left(\phi= \pm 90^{\circ}\right.$; Fig. $\left.5 \mathrm{C}\right)$ was fitted to a Gaussian with a linear background.

The extracted monolayer coverage $C$, relative to a spot below the meniscus edge, is plotted as a function of the spot height $h$ in Fig. 7B, for both before (circles) and after (squares) the LB deposition. The $h$-dependent intensity correction factors used to extract $C$ [Eq. (18)] are plotted in Fig. 7A. It can be seen that before the LB deposition, the monolayer coverage below the meniscus edge is essentially uniform $\left(h<h_{\max }\right)$ and drops to zero immediately above the meniscus edge $\left(h>h_{\max }\right)$. After the LB deposition, the coverage below the meniscus is unchanged, but the coverage above the meniscus edge is now nonzero over $\Delta h \sim 4 \mathrm{~mm}$, due to the LB transfer of the monolayer onto the substrate. The extracted $C$ further reveals that the LB transfer is only partial, indicating that the coverage on the Si substrate is about $75 \%$ of the coverage on the water surface.

IUCr macros version 2.1.6: 2014/10/01 
The above results illustrate the utility of TSAXS in mapping out quantitative information about local liquid-surface structures as a function of position along the surface. Significantly, the results also demonstrate that the utilized experimental approach, based on concurrent TSAXS measurements on meniscus and substrate interfaces, is well suited to enabling in situ structural characterization of the LB transfer process. Indeed, the present work represents a rare study in which in situ x-ray scattering was used to characterize both Langmuir and LB monolayers while they are still in contact. Previously, Durbin et al. used in situ GIXD to characterize the in-plane structure of fatty-acid LB monolayers immediately after the LB deposition (Durbin et al., 1997). The present meniscus-based TSAXS approach has the advantage of being a local structural probe and technically simple to implement.

\subsection{Monolayers of fluorinated fatty acid (C14F)}

The formation of a $\mathrm{C} 14 \mathrm{~F}$ monolayer was first verified by XR and GIXD measurements on a horizontal part of the water surface (Fig. 8). The fitting of a boxmodel electron density profile (Als-Nielsen et al., 1994) to the measured XR data (Fig. 8A) reveals a dense, $2 \mathrm{~nm}$ thick layer (Fig. 8B), consistent with closely packed C14F molecules standing up normal to the interface (Jacquemain et al., 1990). The observed GIXD pattern (Fig. 8C) exhibits Bragg peaks at $q_{x y}=G_{1}=1.25 \AA^{-1}$ and $q_{x y}=\sqrt{3} G_{1}=2.17 \AA^{-1}$, consistent with a hexagonally packed monolayer of C14F chains with a nearest-neighbor $(\mathrm{NN})$ distance of $(2 / \sqrt{3}) 2 \pi / G_{1}=5.8 \AA$. The Bragg-rod intensity (Fig. 8D) is peaked at $q_{z} \approx 0$, indicating that the chains are untilted. The box-model fit to the Bragg rod is consistent with the presence of an ordered layer with

a thickness of $17 \AA$, which agrees well with the tail length of $14 \times 1.25=17.5 \AA$. The observed packing structure is consistent with those reported previously for fluorinated fatty-acid monolayers on water (Barton et al., 1992; Goldmann et al., 1993; Acero

IUCr macros version 2.1.6: 2014/10/01 
et al., 1993).

After these control measurements, TWAXS measurements were carried out on the same $\mathrm{C} 14 \mathrm{~F}$ monolayer at the substrate-supported meniscus-air interface, with the incident X-ray beam normal to the vertical substrate $(\omega=0)$. Typical TWAXS images collected at various $h$ and the corresponding 1D intensity profiles along the horizon $\left(\phi=90^{\circ}\right)$ are summarized in Figs. 9A and 10, respectively. The TWAXS patterns from spots below the meniscus edge $\left(h<h_{\max }\right)$ show the first-order Bragg ring at $q_{x y}=$ $G_{1}=1.25 \AA^{-1}$ as well as a broad ring at higher $q$ due to the liquid structure factor of bulk water (peaked at $q=2.0 \AA^{-1}$ (Hura et al., 2000; Vaknin et al., 2008)). As the beam spot was lowered, the increased x-ray path through the meniscus water resulted in an enhanced background due to the bulk-water scattering (Fig. 10A); however, when this background is subtracted, the Bragg peak from the $\mathrm{C} 14 \mathrm{~F}$ monolayer is clearly discernible (Fig. 10B).

4.2.1. Coverage by ordered domains vs. $h$. The $h$-dependent TWAXS measurements on $\mathrm{C} 14 \mathrm{~F}$ monolayers were carried out both before and after LB deposition onto the vertical substrate (Fig. 9). As in the case of AuNP monolayers, the surface coverage by the ordered $\mathrm{C} 14 \mathrm{~F}$ monolayer was extracted from the observed Bragg-peak intensities using the procedure described in Sec. 4.1.2. The results are summarized in Fig. 11. Interestingly, the calculated total intensity correction factor (Fig. 11A) remains close to unity even below the meniscus edge $\left(h<h_{\max }\right)$. This is due to the particular x-ray energy used for these measurements. The meniscus shape is such that for $13.5 \mathrm{keV}$ $\mathrm{x}$-rays, the intensity-reducing effect of the x-ray absorption through water is canceled just right by the intensity-enhancing effect of the surface tilt, practically over the entire range of $h>0$.

The extracted monolayer coverage shows an expected $h$-dependent behavior (Fig.

IUCr macros version 2.1.6: 2014/10/01 
11B). Before the LB deposition, the monolayer coverage is uniform below the meniscus edge $\left(h<h_{\max }\right)$ but drops to zero above the edge $\left(h>h_{\max }\right)$. After the LB transfer, the monolayer coverage is still uniform for $h<h_{\max }$ but is nonzero for $h>h_{\max }$ over the transfer distance of $\Delta h=4.5 \mathrm{~mm}$, corresponding to the LB monolayer-coated region on the substrate. The LB coverage is only about $70 \%$ relative to the coverage on water, similar to the case of the AuNP monolayers.

4.2.2. Extraction of Bragg rods. A key advantage of grazing incidence scattering methods is that they are well suited to probing Bragg rods (Fig. 8D), whose $q_{z}$ dependence reveals the normal-to-layer structural features of the laterally ordered domains. In this section, we demonstrate that Bragg rods can also be extracted by performing TXS measurements at non-normal incidence.

For simplicity, we consider the case of a vertical, substrate-supported LB monolayer that is rotated about the $z^{\prime}$ axis to impose non-normal incidence, i.e., $\theta=0$ and $\omega \neq 0$ (Sec. 2.2.2). Under this condition, the $\phi$-dependence of the peak position in $q$ is described by Eq. (16). Along this "Bragg ring," $q_{z}$ remains constant if $\omega=0$ but varies with $\phi$ if $\omega \neq 0$, according to Eq. (15). This behavior is demonstrated in Fig. $12 \mathrm{D}$, which contrasts two cases, $\omega=0$ and $9^{\circ}$, for the first-order peak for the $\mathrm{C} 14 \mathrm{~F}$ monolayer $\left(G=1.25 \AA^{-1}\right)$. This plot reveals a few notable features. First, at normal incidence $(\omega=0)$, the constant $q_{z}$ value is nonzero $\left[q_{z} \approx-G^{2} / 2 k=-0.07 \AA^{-1}\right.$ via Eqs. (6), (15), and $q \approx G$ for $\zeta=0.056 \ll 1$ for $22.1 \mathrm{keV}$ x-rays used here]. Second, at non-normal incidence $(\omega \neq 0)$, both positive and negative values of $q_{z}$ can occur. Finally, the larger the value of $\omega$ is, the greater the accessible range of $q_{z}$ is, where the $q_{z}$ range is limited by the values at $\phi= \pm \pi / 2$.

The above analysis implies that a single TXS image taken at $\omega \neq 0$ can reveal the $q_{z}$-dependent intensity variation along the Bragg rod. To demonstrate this, Fig. 12

IUCr macros version 2.1.6: 2014/10/01 
compares TWAXS images taken from the LB monolayer at $\omega=0$ and $9^{\circ}$. For $\omega=0$ (Fig. 12B), the image shows a uniform intensity distribution along the Bragg ring, as expected from the constancy of $q_{z}$ with varying $\phi$ and the 2D powder nature of the ordered monolayer domains. By contrast, for $\omega=9^{\circ}$ (Fig. 12C), the observed intensity distribution is no longer uniform, consistent with $q_{z}$ varying with $\phi$. The $\phi$-dependent variation of the background-subtracted peak intensity for $\omega=9^{\circ}$ is shown in Fig. 12E.

The Bragg rod can be obtained by relating the measured background-subtracted intensity to $\left|q_{z}\right|$, through their $\phi$ dependence (Fig. 12D and E). The resulting TWAXSderived Bragg rod is plotted in Fig. 13 (circles), together with the GIXD-based Bragg rod (triangles). As expected for 2D-powder samples, the TXS-derived data set is noisier than the grazing-incidence data due to the much smaller area of the illuminated sample footprint that contributes to TXS. Nevertheless, the good agreement between the two demonstrates that a single-shot TXS image at non-normal incidence can be used to probe Bragg rods.

\section{Outlook}

The present study can be extended in numerous ways. Possible future directions include: applications of scanning TXS to characterize heterogeneous monolayer structures at liquid surfaces (e.g., morphologies of anisotropic domains in single-phase monolayers, phase coexistence in single- and multi-component monolayers); improvement of spatial resolutions for scanning TXS; in situ TXS studies of LB transfer

processes; improvement of sample throughput and use of TXS as a screening tool to efficiently explore large parameter spaces (e.g., effects of surface and subphase compositions); and investigations into radiation-damage effects and reduction of background scattering.

It is possible to pursue some of these future directions by exploiting the meniscus IUCr macros version 2.1.6: 2014/10/01 
geometry used in this work. However, the meniscus approach clearly has its limitations, with a few notable disadvantages. First, the analysis of TXS data is, although tractable as shown above, complicated somewhat by the surface curvature and the $h$-dependent variations in the x-ray beam path through bulk liquid. Second, due to the need for a vertical substrate, the meniscus approach is not well suited to accommodating a large enough array of samples to significantly improve sample throughput. Finally, due to the need to move the sample rather than the beam, achievable spatial resolutions for scanning-probe TXS may be limited by motion-induced surface disturbance rather than by the beam size, unless the surface structure is solid-like and fully covers the available surface.

To address these issues, another worthwhile future direction may be to develop a vertical scattering geometry, together with development of an optimized samplecell setup, such that TXS measurements can be performed on flat, horizontal liquid surfaces. As compared to the meniscus approach, vertical TXS on horizontal liquid surfaces should offer simpler analysis geometry and be better suited to accommodating a $2 \mathrm{D}$ array of samples to increase throughput. The simplest approach to implement a vertical scattering geometry is to utilize a Bragg-reflecting crystal to deflect the $\mathrm{x}$-ray beam upward such that the incident beam strikes the horizontal liquid surface from below, as shown in Fig. 14A. This can be facilitated by choosing a crystal ( $\mathrm{X}$ in Fig. 14A) with a Bragg angle $2 \theta_{B} \sim 90^{\circ}$ (e.g., Ge(555) for x-rays at $13.5 \mathrm{keV}$ ). The sample cell may consist of a liquid well with a thin, subsurface window that the liquid can wet (e.g., a hydrophilic window for water), in order to let the incident beam through and limit the liquid thickness at the illuminated spot (e.g., to a few hundred microns for water). TXS patterns can be collected by a downward-facing area detector positioned above the sample surface. For scanning-probe measurements, the sample can remain stationary, and the beam spot on the surface can be controlled by a translation and a

IUCr macros version 2.1.6: 2014/10/01 
rotation of the steering crystal (Fig. 14A), where the crystal is rotated about the axis of the incoming beam to maintain the Bragg condition. The main disadvantages of this approach, however, are that it would require a specific combination of a steering crystal plane and x-ray energy and that the rotation of the crystal changes both the beam spot position and the incident angle on the liquid surface.

An alternative approach that is more versatile, but also technically more challenging, is to couple the above vertical scattering geometry to a conventional liquid-surface diffractometer with tunable x-ray energy, as shown in Fig. 14B. A liquid-surface diffractometer is equipped with a beam-steering crystal (X1) which controls the angle $\alpha$ $\left( \pm 10^{\circ}\right)$ for the downward (or upward) deflection of the synchrotron-generated, horizontal x-ray beam (Pershan \& Schlossman, 2012). In this scheme, a vertically deflecting crystal X2 is mounted on the liquid-surface diffractometer to Bragg-reflect the beam from X1 up toward the horizontal liquid surface, positioned above X2. This should enable precise control of the incident beam direction relative to the liquid surface through adjustments of $\alpha$ and the x-ray energy, which sets $\theta_{B}$. Moreover, by translating the two steering crystals X1 and X2, it should be possible to continually shift the x-ray-illuminated spot along a stationary liquid surface, while keeping the incident angle fixed.

\section{Summary}

To test the feasibility of using TXS as a probe for complex liquid surface structures, we performed TXS measurements on Langmuir films formed at the surface of a welldefined water meniscus, induced by complete wetting of a vertical, hydrophilic Si substrate. A first-order peak corresponding to $2 \mathrm{D}$ hexagonal packing was clearly observed in the measured TSAXS patterns from an AuNP monolayer $\left(q_{x y}=0.07 \AA^{-1}\right)$ and the TWAXS patterns from a C14F monolayer $\left(q_{x y}=1.25 \AA^{-1}\right)$. For both systems, the LB 
transfer of the monolayer onto the vertical Si substrate allowed us to apply in situ TXS on both the LM at the meniscus-air interface and the LB monolayer. The results of position-dependent TXS measurements were analyzed to extract the meniscus shape and the variations in the monolayer coverage across the meniscus edge, i.e., across the LM-LB boundary. Our results strongly support the utility of scanning TXS as a practical probe for position-dependent structural charcterizations at liquid surfaces, with submillimeter resolutions. We have also shown that TXS at non-normal incidence can be used to probe Bragg rods.

These results, together with continual improvement in the brightness and spot size of x-ray beams available at synchrotron facilities, raise the possibility of realizing scanning transmission x-ray scattering microscopy (STXSM) for structures at liquid surfaces. Successful development of STXSM will enable simultaneous measurements of nanoscale packing structure and morphologies of microns-to-submillimeter scale domains at the interface. Use of TXS as a high-throughput screening tool for characterizing liquid-surface structures represents another promising direction for future development.

The BNL contribution to this work was supported by the U. S. Department of Energy, Basic Energy Sciences, by the Materials Sciences and Engineering Division (M. F.), through the National Synchrotron Light Source II (M. F. and L. Y.), and through the Center for Functional Nanomaterials (D. N.), under contract no. DEAC02-98CH10886. Use of the National Synchrotron Light Source was supported by the U. S. Department of Energy, Office of Basic Energy Sciences, under contract no. DE-AC02-98CH10886. This research used resources of the Center for Functional Nanomaterials, which is a U. S. DOE Office of Science Facility, at Brookhaven National Laboratory under Contract No. DE-SC0012704. The work by I. K. and use of the Advanced Photon Source were supported by the U. S. Department of Energy, Office 
of Basic Energy Sciences, under contract no. DE-AC02-06CH11357.

\section{References}

Acero, A. A., Li, M., Lin, B., Rice, S. A., Goldmann, M., Azouz, I. B., Goudot, A. \& Rondelez, F. (1993). J. Chem. Phys. 99, 7214-7220.

Als-Nielsen, J., Christensen, F. \& Pershan, P. S. (1982). Phys. Rev. Lett. 48, 1107-1110.

Als-Nielsen, J., Jacquemain, D., Kjaer, K., Leveiller, F., Lahav, M. \& Leiserowitz, L. (1994). Phys. Rep. 246, 251-313.

Barton, S. W., Goudot, A., Bouloussa, O., Rondelez, F., Lin, B., Novak, F., Acero, A. \& Rice, S. A. (1992). J. Chem. Phys. 96, 1343-1351.

Danauskas, S. M., Ratajczak, M. K., Ishitsuka, Y., Gebhardt, J., Schultz, D., Meron, M., Lin, B. H. \& Lee, K. Y. C. (2007). Rev. Sci. Instrum. 78, 103705.

Durbin, M. K., Malik, A., Richter, A. G., Huang, K. G. \& Dutta, P. (1997). Langmuir, 13, $6547-6549$.

Fradin, C., Braslau, A., Luzet, D., Smilgies, D., Alba, M., Boudet, N., K. Mecke, K. \& Daillant, J. (2000). Nature, 403, 871-874.

Fukuto, M., Nguyen, Q. L., Vasilyev, O., Mank, N., Washington-Hughes, C. L., Kuzmenko, I., Checco, A., Mao, Y., Wang, Q. \& Yang, L. (2013). Soft Matter, 9, 9633-9642.

Fukuto, M., Wang, S. T., Lohr, M. A., Kewalramani, S. \& Yang, L. (2010). Soft Matter, 6, 1513-1519.

de Gennes; F. Brochard-Wyart; P.-G. \& Quere, D. (2004). Capillarity and Wetting Phenomena. New York: Springer.

Goldmann, M., Nassoy, P. \& Rondelez, F. (1993). Physica A, 200, 688-695.

Hiramatsu, H. \& Osterloh, F. E. (2004). Chem. Mater. 16, 25092511.

Hura, G., Sorenson, J., Glaeser, R. \& Head-Gordon, T. (2000). J. Chem. Phys. 113, 91409148 .

Jacquemain, D., Wolf, S. G., Leveiller, F., Lahav, M., Leiserowitz, L., Deutsch, M., Kjaer, K. \& Als-Nielsen, J. (1990). J. Am. Chem. Soc. 112, 7724-7736.

Kaganer, V. M., Mohwald, H. \& Dutta, P. (1999). Rev. Mod. Phys. 71, 779-819.

Kerschnitzki, M., Kollmannsberger, P., Burghammer, M., Duda, G. N., Weinkamer, R., Wagermaier, W. \& Fratzl, P. (2013). J. Bone Miner. Res. 28, 18371845.

Kewalramani, S., Wang, S. T., Lin, Y., Nguyen, H. G., Wang, Q., Fukuto, M. \& Yang, L. (2011). Soft Matter, 7, 939-945.

Kim, F., Kwan, S., Akana, J. \& Yang, P. D. (2001). J. Am. Chem. Soc. 123, 43604361.

Kuzmenko, I., Rapaport, H., Kjaer, K., Als-Nielsen, J., Weissbuch, I., Lahav, M. \& Leiserowitz, L. (2001). Chem. Rev. 101, 1659-1696.

Luo, G. M., Malkova, S., Yoon, J., Schultz, D. G., Lin, B. H., Meron, M., Benjamin, I., Vanysek, P. \& Schlossman, M. L. (2006). Science, 311, 216-218.

Narayanan, S., Wang, J. \& Lin, X.-M. (2004). Phys. Rev. Lett. 93, 135503.

Ocko, B. M., Wu, X. Z., Sirota, E. B., Sinha, S. K., Gang, O. \& Deutsch, M. (1997). Phys. Rev. E, 55, 3164-3182.

Paris, O. (2008). Biointerphases, 3, FB16-FB26.

Pershan, P. S. (2014). J. Appl. Phys. 116, 222201.

Pershan, P. S. \& Schlossman, M. L. (2012). Liquid Surfaces and Interfaces: Synchrotron X-ray Methods. Cambridge, UK: Cambridge University Press.

Ratanabanangkoon, P. \& Gast, A. (2003). Langmuir, 19, 1794-1801.

Sanyal, M. K., Sinha, S. K., Huang, K. G. \& Ocko, B. M. (1991). Phys. Rev. Lett. 66, 628-631.

Schwartz, D. K., Schlossman, M. K., Kawamoto, E. H., Kellogg, G. J., Pershan, P. S. \& Ocko, B. M. (1990). Phys. Rev. A, 41, 5687-5690.

Seidel, R., Gourrier, A., Burghammer, M., Riekel, C., Jeronimidis, G. \& Paris, O. (2008). Micron, 39, 198205.

IUCr macros version 2.1.6: 2014/10/01 
Srivastava, S., Nykypanchuk, D., Fukuto, M. \& Gang, O. (2014a). ACS Nano, 8, 9857-9866.

Srivastava, S., Nykypanchuk, D., Fukuto, M., Halverson, J. D., Tkachenko, A. V., Yager, K. G. \& Gang, O. (2014b). J. Am. Chem. Soc. 136, 8323-8332.

Stanley, J., Boucheron, L., Lin, B. H., Meron, M. \& Shpyrko, O. (2015). Appl. Phys. Lett. 106, 161602.

Tao, A. R., Huang, J. X. \& Yang, P. D. (2008). Acc. Chem. Res. 41, 16621673.

Vaknin, D., Bu, W. \& Travesset, A. (2008). J. Chem. Phys. 129, 044504.

Vaknin, D., Kruger, P. \& Losche, M. (2003). Phys. Rev. Lett. 90, 178102.

Wang, S.-W., Poglitsch, C. L., Yatcilla, M. T., Robertson, C. R. \& Gast, A. P. (1997). Langmuir, 13, 57945798.

Wang, X., Xu, S., Zhou, J. \& Xu, W. (2010). J. Colloid Interface Sci. 348, 24-28.

Weaver, J. C., Milliron, G. W., Miserez, A., Evans-Lutterodt, K., Herrera, S., Gallana, I., Mershon, W. J., Swanson, B., Zavattieri, P., DiMasi, E. \& Kisailus, D. (2012). Science, 336, $1275-1280$.

Witte, K. N., Kewalramani, S., Kuzmenko, I., Sun, W., Fukuto, M. \& Won, Y.-Y. (2010). Macromolecules, 43, 2990-3003.

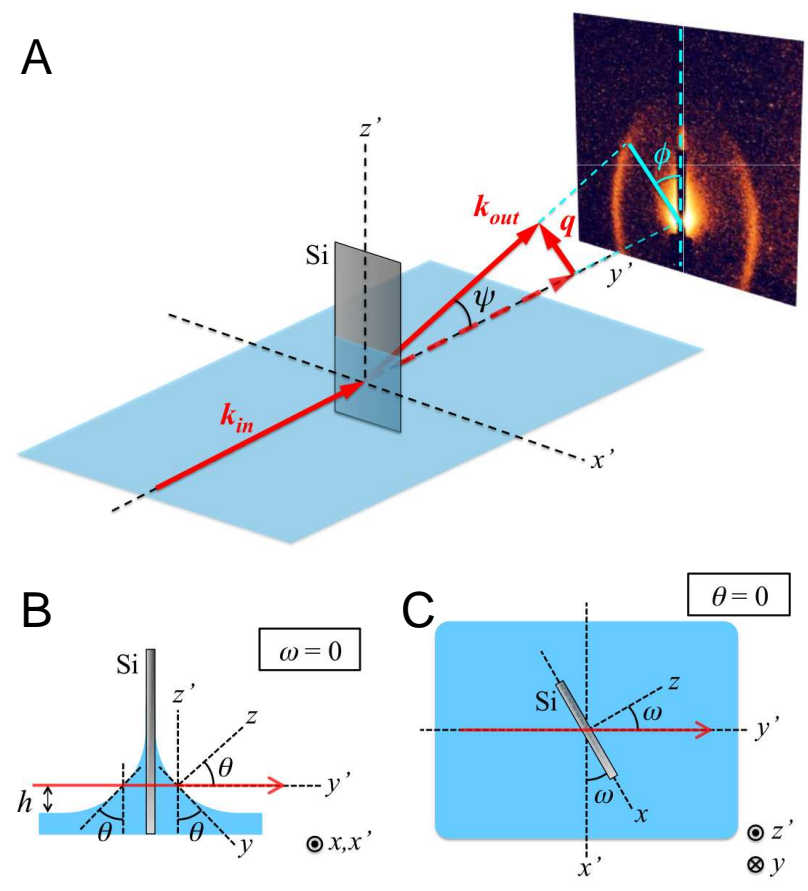

Fig. 1. (A) Schematic illustrating the transmission x-ray scattering geometry used in the present study. (B) A limiting case in which the meniscus-inducing Si substrate is normal to the incident x-ray beam $(\omega=0)$. (C) A limiting case in which the x-ray-illuminated surface is vertical $(\theta=0)$. In $(\mathrm{A})-(\mathrm{C})$, the coordinates $\left(x^{\prime}, y^{\prime}, z^{\prime}\right)$ represent the laboratory frame, with the $z^{\prime}$ axis along the gravity axis and the $y^{\prime}$ axis along the horizontal incident $\mathrm{x}$-ray beam. The coordinates $(x, y, z)$ are defined with respect to the local surface at the x-ray-illuminated spot, with the $z$ axis along the surface normal. 


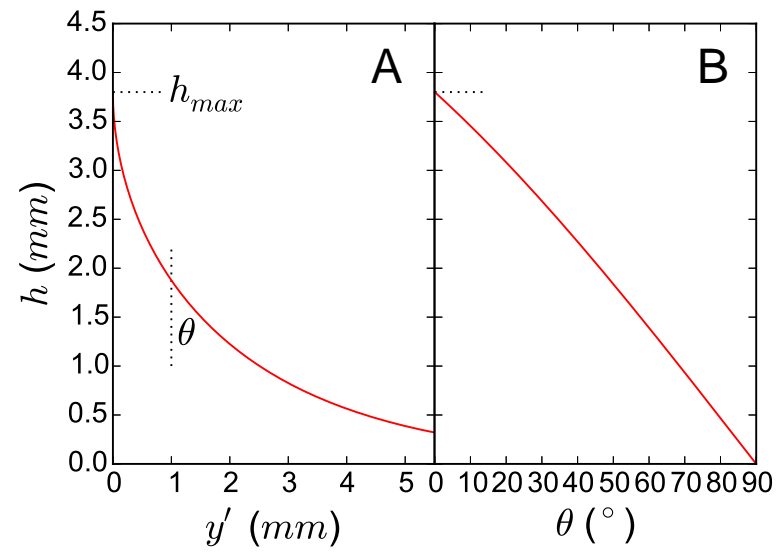

Fig. 2. Expected meniscus shape of water completely wetting a vertical, hydrophilic wall. (A) Local height $h$ of meniscus surface as a function of the lateral distance $y^{\prime}$ from the wall, normal to the substrate. (B) Relation between $h$ and the local surface tilt angle $\theta$, defined such that it reduces to the contact angle $\theta=0$ at the meniscus edge $h=h_{\max }$.
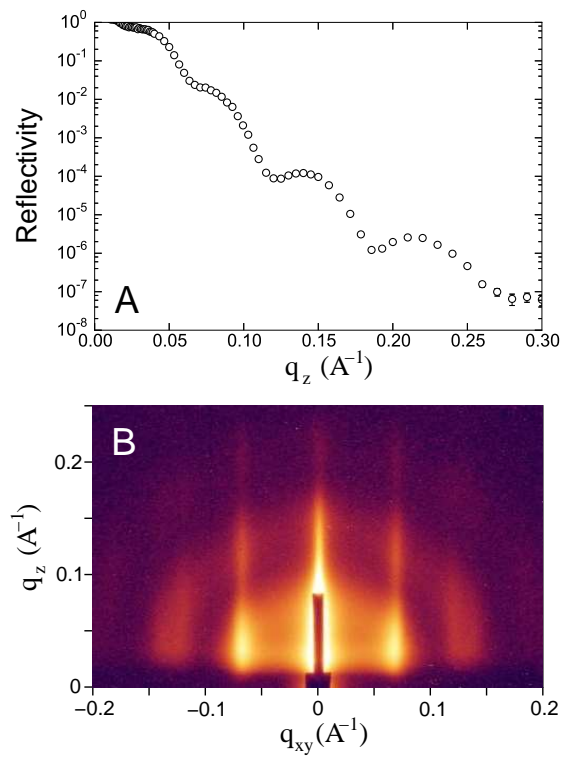

Fig. 3. (A) Measured XR and (B) GISAXS image from a Langmuir monolayer of AuNPs at the air-water interface, from a spot on the flat horizontal surface located far from the vertically immersed Si substrate. 

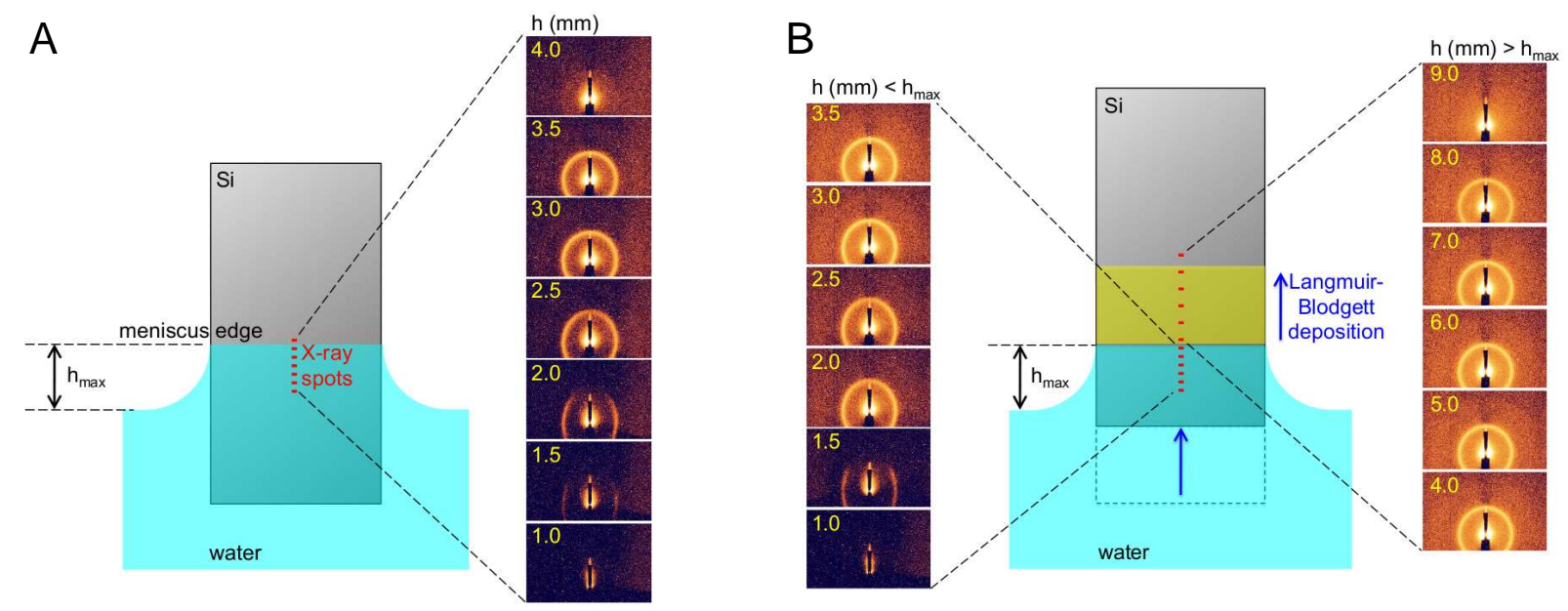

Fig. 4. (A) TSAXS images from Langmuir monolayer of AuNPs at the air-water meniscus interface against a vertically immersed Si substrate. (B) TSAXS images measured after raising the Si substrate by $4.5 \mathrm{~mm}$, leading to Langmuir-Blodgett deposition of the AuNP monolayer onto the substrate. 

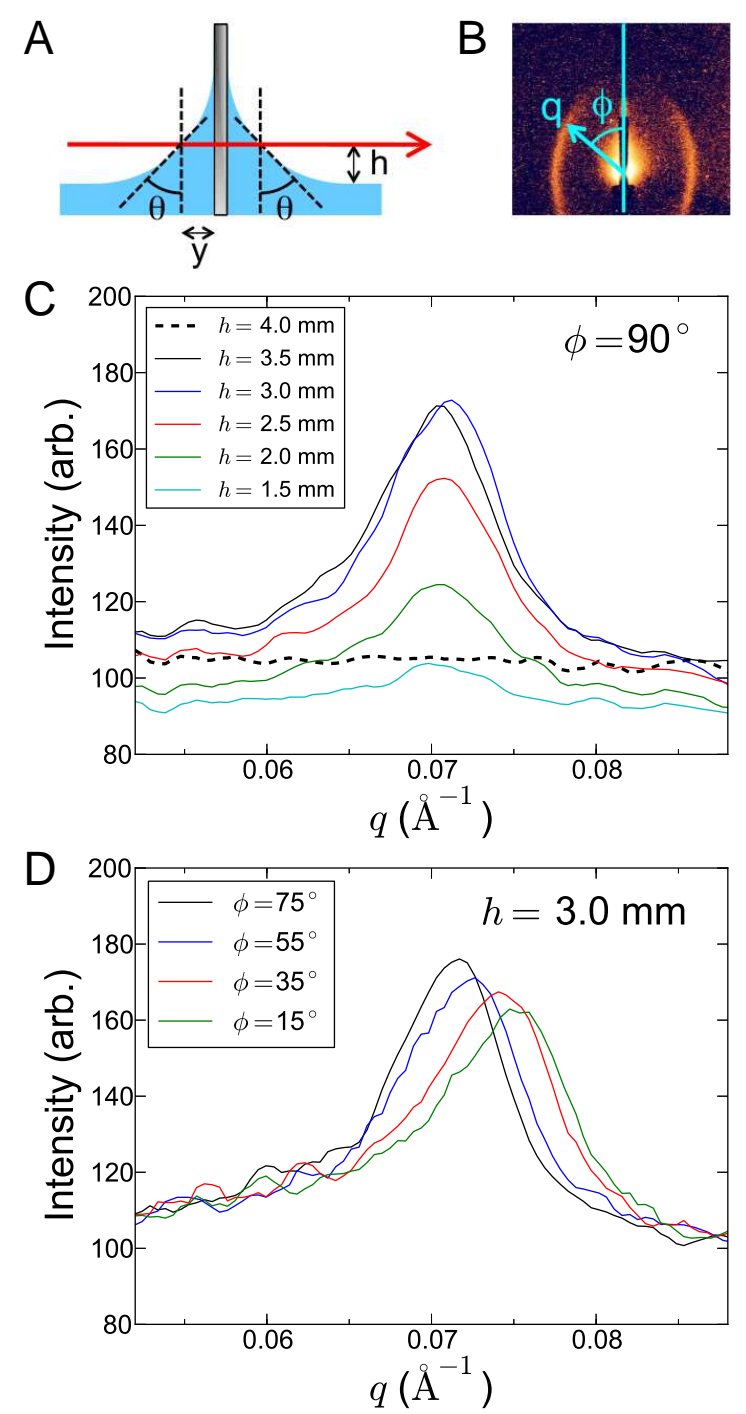

Fig. 5. (A) Schematic illustrating the relations between the local meniscus-surface height $h$, tilt $\theta$, and distance $y^{\prime}$ from the substrate, where the incident x-ray beam is normal to the substrate surface $(\omega=0)$. (B) Definition of the wave-vector azimuthal angle $\phi$ in the detector plane. (C) Observed 1D TSAXS intensity profiles as a function of $q$ along the horizon $(\phi=\pi / 2)$, from Langmuir monolayers of AuNP at various $h$ along the water meniscus surface (before LB transfer). (D) Observed 1D intensity profiles along different azimuthal $(\phi)$ directions, from a AuNP monolayer on meniscus surface at $h=3.0 \mathrm{~mm}$. 

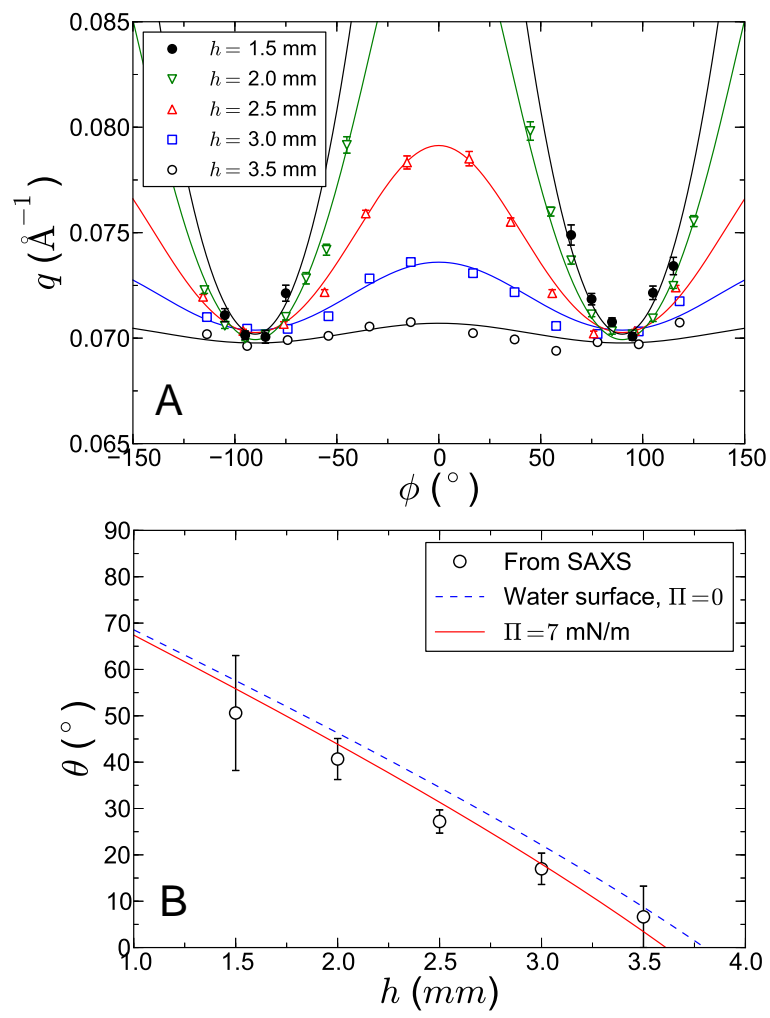

Fig. 6. (A) The $\phi$ dependence of the total wavevector $q$ for the first Bragg condition based on the TSAXS images from Langmuir monolayer of gold NPs at the air-water meniscus interface against a vertically immersed Si substrate. The extracted data (symbols) and best fits (lines) are shown for x-ray spots at heights $h=1.5,2.0$, 2.5, 3.0, and $3.5 \mathrm{~mm}$ above the horizontal water surface. (B) Extracted surface tilt $\theta$ (symbols) as a function of x-ray spot height $h$. The lines represent predictions, based on Eq. (2), for bare water surface (dashed line) and for the surface pressure of $\Pi=7 \mathrm{mN} / \mathrm{m}$ (solid line), at which the TSAXS images were taken. 

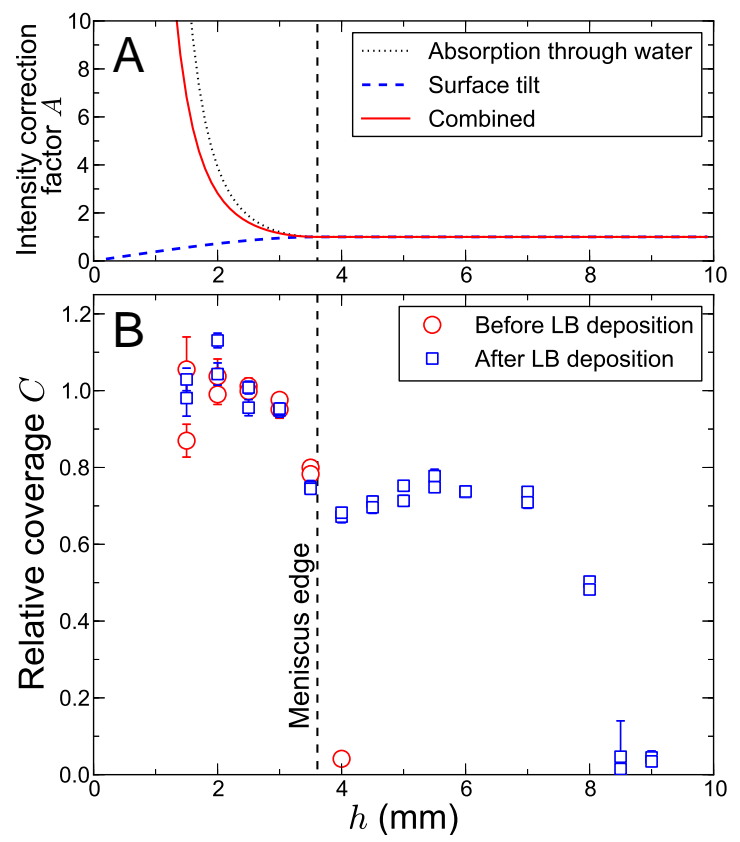

Fig. 7. TSAXS-derived surface coverage by ordered domains of AuNP monolayers, as a function of local surface height $h$. (A) Calculated TSAXS intensity correction factors, for x-ray absorption through bulk meniscus water (dotted line; for $8.17 \mathrm{keV}$ $\mathrm{x}$-rays used), surface tilt (dashed line), and the product of the two (solid line). (B) Normalized surface coverage extracted from the background-subtracted, meniscuscorrected peak TSAXS intensities observed at the horizon $(\phi= \pm \pi / 2)$, before (circles) and after (squares) LB deposition. The coverage for $h<h_{\max }$ corresponds to Langmuir monolayers at the air-meniscus water interface; the coverage for $h>$ $h_{\max }$ is for LB monolayers transferred onto the Si substrate. 

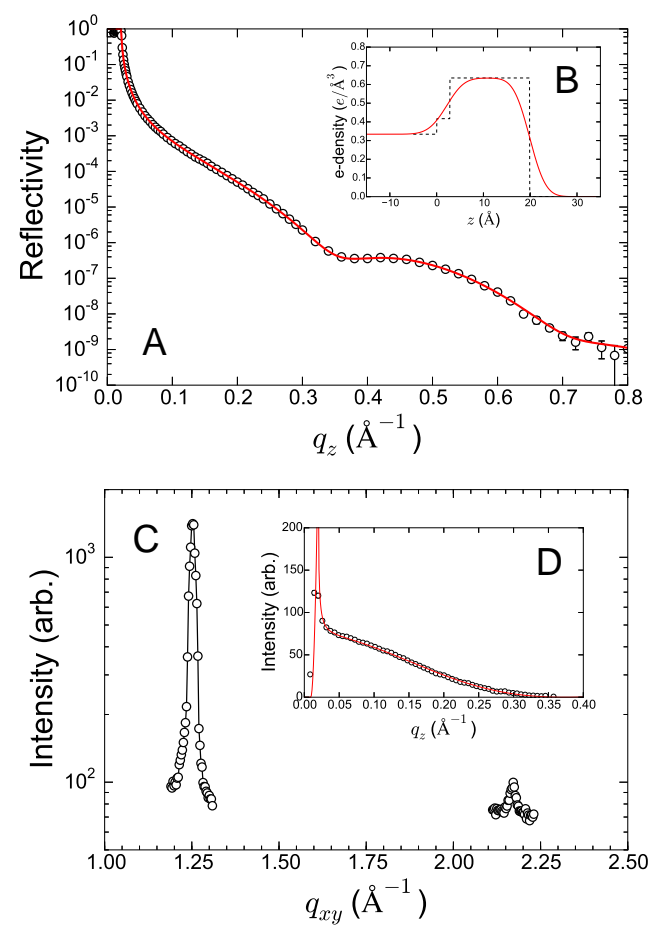

Fig. 8. Data from a Langmuir monolayer of $\mathrm{C} 14 \mathrm{~F}$ at the air-water interface: (A) XR data (circles) and a fit (line) based on a box-model electron density profile, shown in (B); (C) GIXD data; and (D) Bragg rod data (circles) for the first-order peak at $q_{x y}=1.25 \AA^{-1}$. The line is a box-model fit of form $I\left(q_{z}\right) \propto T\left(q_{z}\right)\left[\operatorname{sinc}\left(q_{z} L / 2\right)\right]^{2}$ with a layer thickness of $L=16.8 \AA$, where $T\left(q_{z}\right)$ is the surface enhancement factor (Jacquemain et al., 1990) and $\operatorname{sinc}(z)=\sin (z) / z$. 


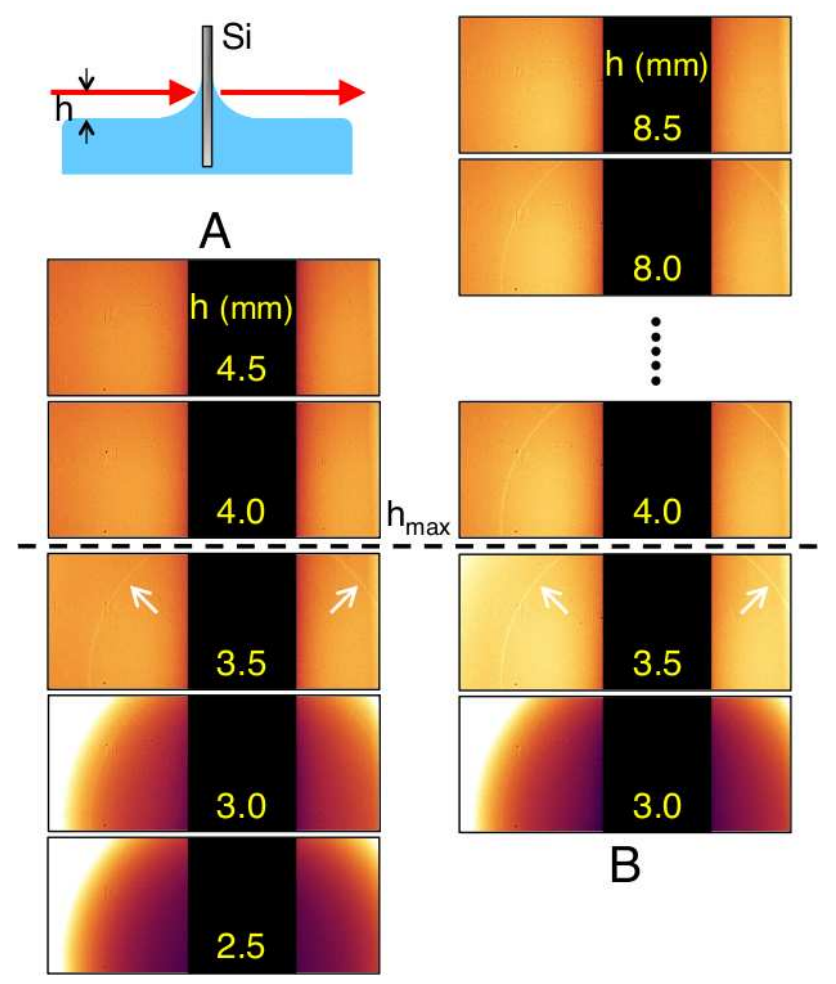

Fig. 9. (A) TWAXS images from Langmuir monolayer of $\mathrm{C} 14 \mathrm{~F}$ at the air-water meniscus interface against a vertically immersed Si substrate. (B) TWAXS images after raising the Si substrate by $4.5 \mathrm{~mm}$, leading to Langmuir-Blodgett deposition of the $\mathrm{C} 14 \mathrm{~F}$ monolayer onto the substrate. The arrows in the images for $h=3.5 \mathrm{~mm}$ indicate the first-order diffraction peak at $q=1.25 \AA^{-1}$ from the $\mathrm{C} 14 \mathrm{~F}$ monolayer. The dashed horizontal line separates the images taken above and below the meniscus edge $\left(h_{\max }\right)$. The diffuse ring at large angles whose intensity increases with decreasing $h$ arises from the liquid structure factor of the bulk water in the meniscus. 

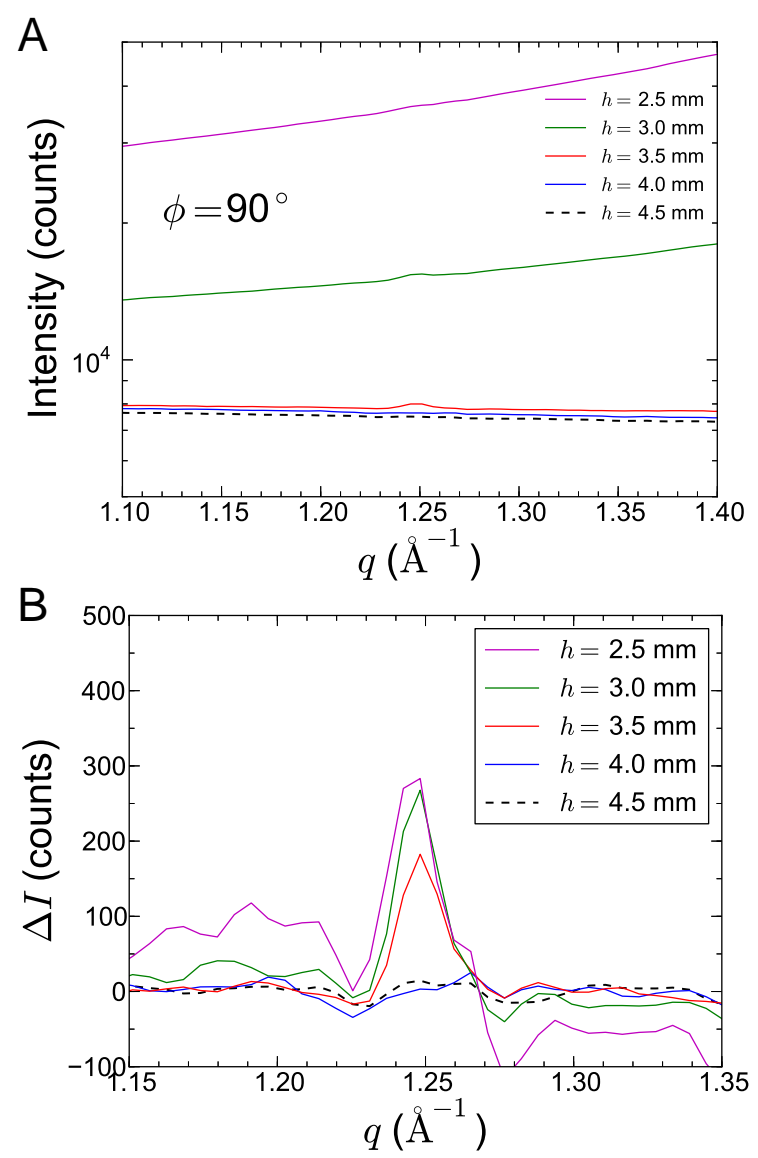

Fig. 10. Observed 1D TWAXS intensity profiles as a function of $q$ along the horizon $(\phi=\pi / 2)$, from Langmuir monolayers of $\mathrm{C} 14 \mathrm{~F}$ at various $h$ along the water meniscus surface (before LB transfer): (A) raw data; (B) the same data after subtracting linear backgrounds. 


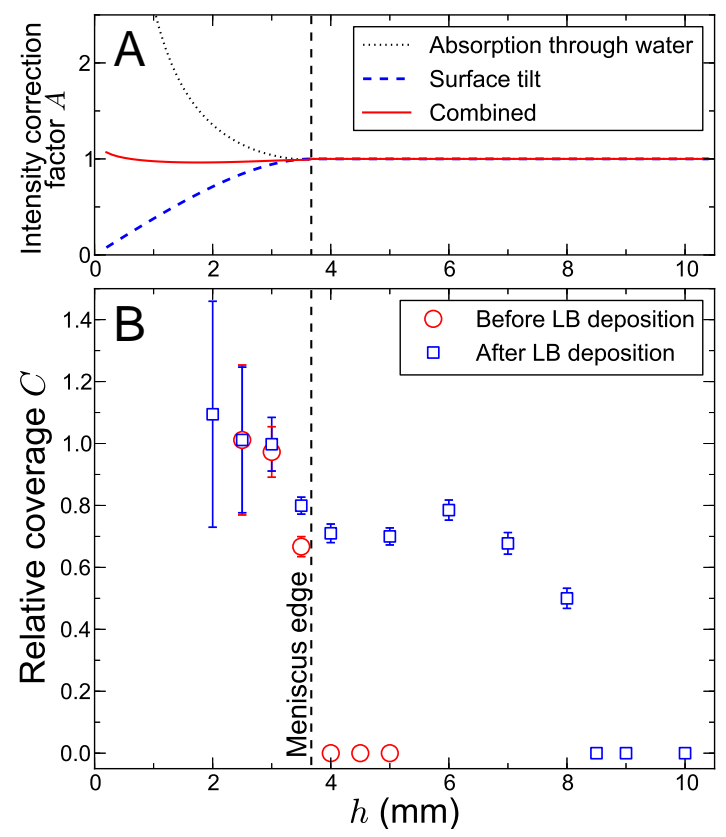

Fig. 11. TWAXS-derived surface coverage by ordered domains of C14F monolayers, as a function of local surface height $h$. (A) Calculated TWAXS intensity correction factors, for x-ray absorption through bulk meniscus water (dotted line; for $13.5 \mathrm{keV}$ x-rays used), surface tilt (dashed line), and the product of the two (solid line). (B) Normalized surface coverage extracted from the background-subtracted, meniscus-corrected peak TWAXS intensities observed at the horizon $(\phi=\pi / 2)$, before (circles) and after (squares) LB deposition. The coverage for $h<h_{\max }$ corresponds to Langmuir monolayers at the air-meniscus water interface; the coverage for $h>h_{\max }$ is for LB monolayers transferred onto the Si substrate. 

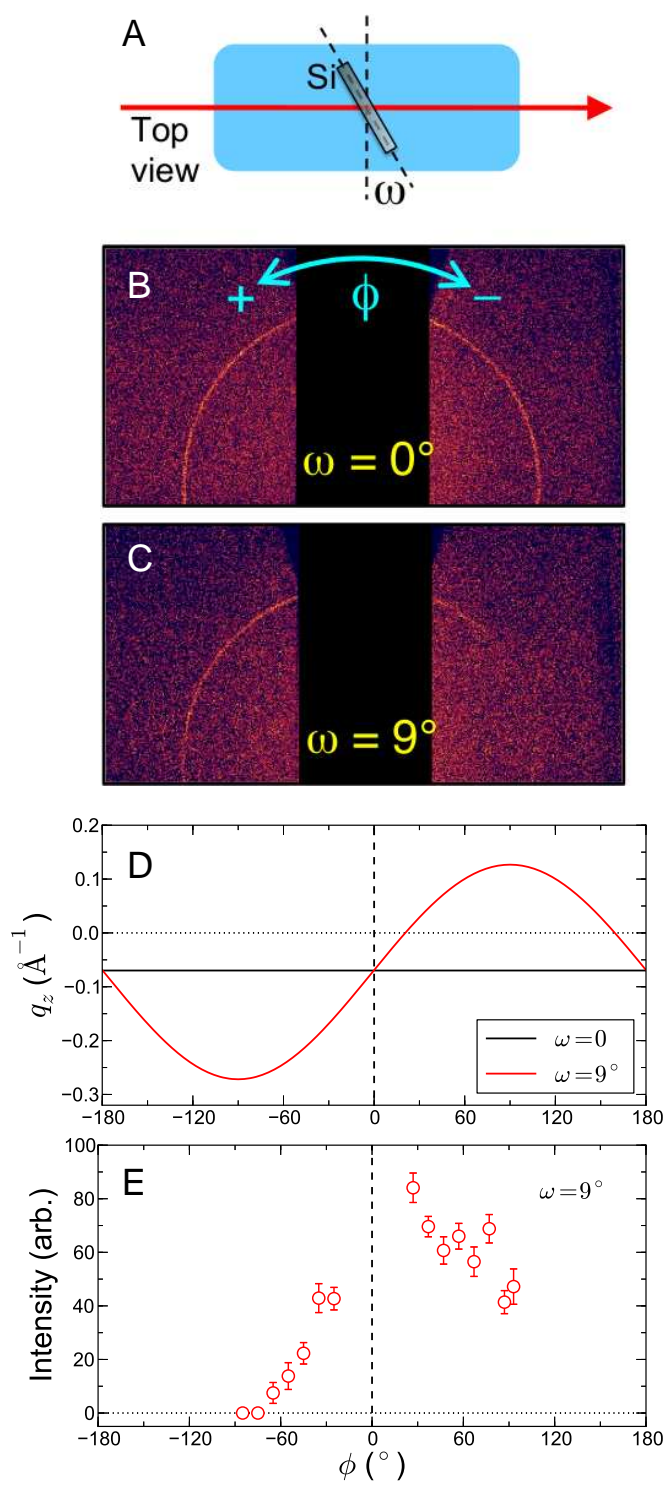

Fig. 12. (A) Definition of the substrate rotation angle $\omega$. Background-subtracted TWAXS images from LB monolayers of $\mathrm{C} 14 \mathrm{~F}$, measured at (B) $\omega=0$ (normal incidence) and (C) $\omega=9^{\circ}$. (D) Expected $\phi$ dependence of $q_{z}$ along the "Bragg ring" at $q_{x y}=1.25 \AA^{-1}$, via Eq. (15), for $\omega=0$ (black) and $9^{\circ}$ (red). (E) Backgroundsubtracted peak intensity for the data at $\omega=9^{\circ}$ in (C) as a function of $\phi$. 


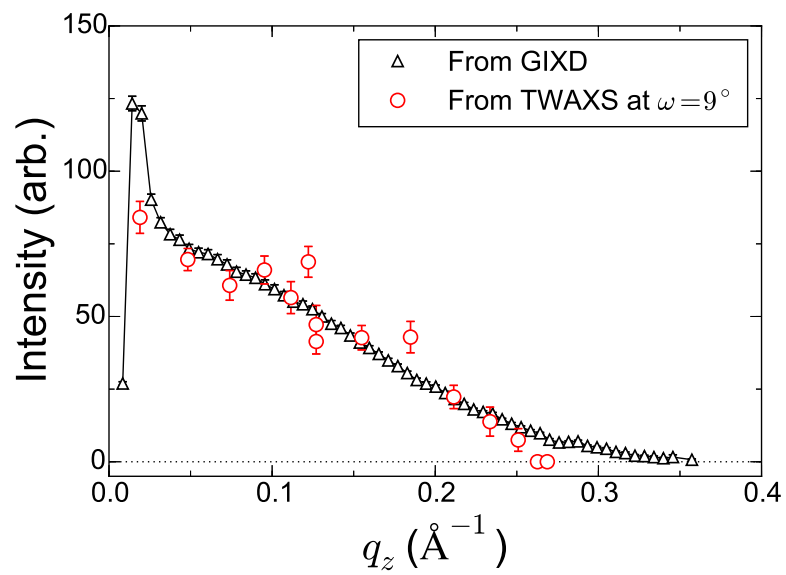

Fig. 13. Measured Bragg rod for the $\mathrm{C} 14 \mathrm{~F}$ monolayer, corresponding to the firstorder, hexagonal-packing peak at $q_{x y}=1.25 \AA^{-1}$. Triangles are from GIXD on a Langmuir monolayer (identical to the data in Fig. 8D). Circles are from TWAXS on a LB monolayer at $\omega=9^{\circ}$. 


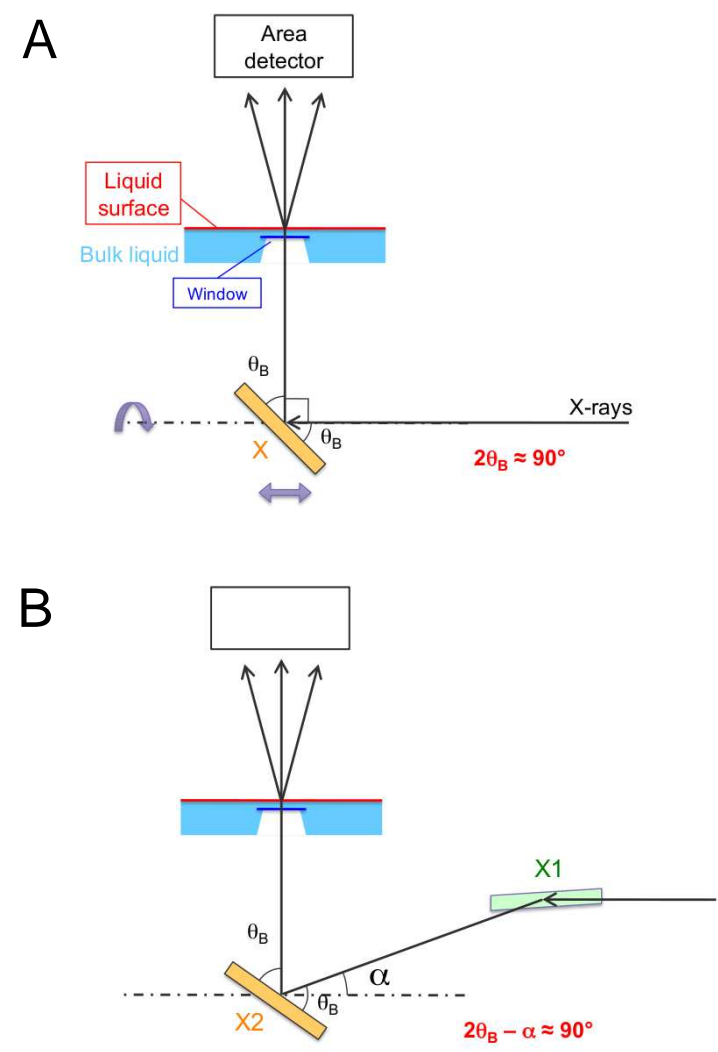

Fig. 14. Schematic illustrations of possible approaches to implement TXS measurements on a flat, horizontal liquid surface. (A) A vertical scattering approach based on a Bragg crystal $(\mathrm{X})$ that deflects a horizontal incoming beam upward, with the Bragg angle $\theta_{B}$ satisfying $2 \theta_{B} \approx 90^{\circ}$. (B) An alternative approach based on the coupling of the vertical scattering geometry to a liquid-surface diffractometer (LSD). Here, the first crystal (X1), part of the LSD, steers the beam to control the angle $\alpha$ to the horizontal (gravity-normal) plane; the second crystal (X2), mounted at the LSD's "sample" position, deflects the beam from X1 upward towards the liquid surface, satisfying $2 \theta_{B}-\alpha \approx 90^{\circ}$.

\section{Synopsis}

The potential of scanning transmission x-ray scattering as a position-sensitive probe for structures at liquid surfaces is explored. 